\section{Enterococcus faecalis ST21 harbouring Tn6009 isolated from a carriage sample in South Africa}

To the Editor: Enterococcus faecalis is an opportunistic pathogen included in the ESKAPE (Enterococcus spp., Staphylococcus aureus, Klebsiella pneumoniae, Acinetobacter baumannii, Pseudomonas aeruginosa and Enterobacter spp.) group that is commonly observed as a commensal in the gastrointestinal tract, but can also opportunistically cause serious infections..$^{[1,2]}$ E. faecalis has been implicated globally in difficult-to-treat infections through acquired multidrug resistance (MDR) as well as the numerous virulence factors it expresses. ${ }^{[3]}$ Several reports have shown that the high virulence and resistance in E. faecalis were associated with pathogenicity islands and mobile genetic elements (MGEs). ${ }^{[2]}$

We report on a virulent, vancomycinresistant E. faecalis (VRE) ST21 (A113R1B0) isolate, carrying several resistance determinants and integrative conjugative elements Tn6009, colonising a patient admitted to a medical ward at a district hospital in uMgungundlovu district, South Africa (SA). The isolate was one of 38 non-duplicate VREs isolated in a larger study on colonisation with ESKAPE pathogens in hospitalised patients in medical and surgical wards. ${ }^{[4]}$ Enterococci from rectal swabs were phenotypically screened for resistance to a panel of antibiotics as previously described. ${ }^{[4]}$ A selected sample of isolates underwent whole-genome sequencing (WGS) and bioinformatics analysis, with Comprehensive Antibiotic Resistance Database (CARD), ResFinder, VirulenceFinder, PlasmidFinder and MGEFinder being used to detect resistance genes, virulence factors, plasmids and MGE.

The isolate carried several resistance genes encoding for resistance to tetracycline (tet $M)$ and macrolides (ermB), as well as the insertion sequence (IS) IsaA encoding efflux systems such as MATE, MFS and $p m r A$ that conferred resistance to lincosamides and streptogramins. A total of 14 putative virulence genes including ace and $e f a A$ genes that are involved in facilitating cell wall adhesion and thereby human pathogenicity were detected. The bacterial sex pheromone genes $c C F 10, C a m E$, $c a d$ and $c O B 1$ that drive the transfer of resistance and virulence genes through horizontal transfer of the plasmid pCF10 among enterococci and other bacteria were additionally detected. The $e b p A, e b p B$ and $e b p C$ genes associated with sortase $(\operatorname{srt} C)$ assist in adherence and biofilm formation and contribute significantly to the pathogenicity of E. faecalis in nosocomial infection. In addition, the thiol peroxidase (tpx), antiphagocytosis (cpsA/uppS, cps $\mathrm{B} / c d s \mathrm{~A})$ were also detected involved in the regulation of oxidant-inducible expression of genes (Table 1). Furthermore, in silico genomic analysis revealed that the isolate harboured a plasmid repUS43 that encodes for the tetracycline resistance gene tet $(M)$ and harbours a highly transmissible non-composite conjugative transposon Tn6009 linked to an $S$. aureus mer and previously identified in in Gram-negative bacteria from Nigeria and Gram-negative and positive bacteria from Portugal. ${ }^{[6]}$ The isolate further harboured an array of MGEs such as CRISPR, phages and insertion sequences, as set out in Table 1 .

VRE is a serious public health threat worldwide, although there is a dearth of information regarding the clonal structure of Enterococcus spp. in Africa. To the best of our knowledge, this is the first report of a VRE ST21 harbouring repUS43 together with Tn6009, cCF10 and tet(M) in SA. A study from Norway revealed that $E$. faecalis ST21 involved in peripheral periodontitis in hospitalised patients harboured rep 9 plasmids and carried Tn916 coupled with tet $(M), \operatorname{erm}(B)$ and integrase genes int $T n \cdot{ }^{[5]}$ Another report showed that $\operatorname{Tn} 6009$ was associated to Tn916like elements in $S$. aureus contributing to the dissemination of MDR determinants that could be transferred from numerous bacteria, including K. pneumoniae, Serratia liquefaciens, Pseudomonas spp. and Streptococcus spp., ${ }^{[6]}$ with the maximum transmissibility rate occurring in Enterococcus spp.

Our findings suggest that the presence of Tn6009 in the microbiome coupled with immunocompromised host status may facilitate transfer of resistance and virulence factors and consequently contribute to the fitness and pathogenicity of commensal bacteria that could subsequently lead to outbreaks not only due to E. faecalis but also other bacteria present in the microbiome. Further molecular studies are warranted to monitor the genomic and pathogenicity trends of both clinical and carriage isolates across geographical locations, in order to prevent outbreak situations. Rational use of antibiotics in community and clinical settings is also essential to curtail antimicrobial resistance emergence and spread.

\section{Raspail Carrel Founou}

Antimicrobial Research Unit, College of Health Sciences, University of KwaZulu-Natal, Durban, South Africa; and Department of MicrobiologyHaematology-Immunology, Faculty of Medicine and Pharmaceutical Sciences, University of Dschang, Cameroon

czangue@yahoo.fr,czangue@gmail.com

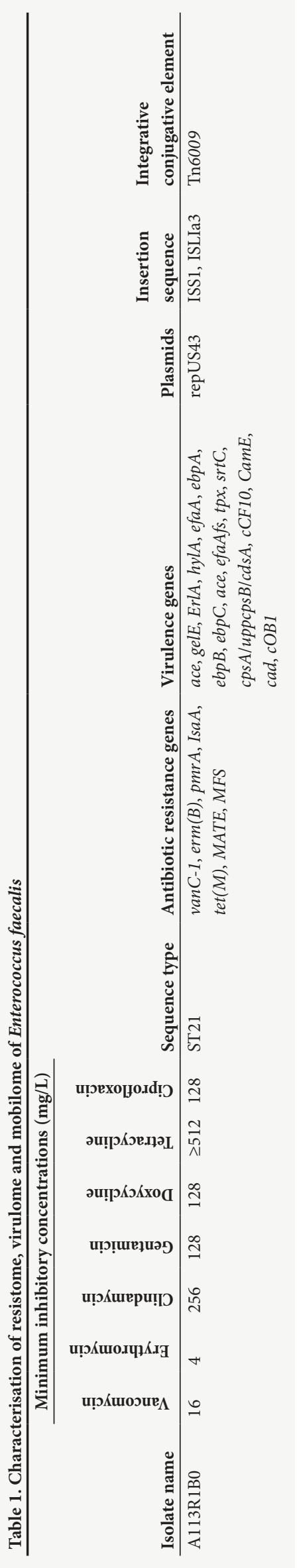




\section{Luria Leslie Founou}

Antimicrobial Research Unit, College of Health Sciences, University of KwaZulu-Natal, Durban, South Africa; Department of Food Safety and Environmental Microbiology, Centre of Expertise and Biological Diagnostic of Cameroon, Yaoundé, Cameroon; Bioinformatics and Applied Machine Learning Research Unit, EDEN Foundation, Yaoundé, Cameroon; and AMR Insights Ambassador Network, Amsterdam, Netherlands

\section{Mushal Allam, Arshad Ismail}

Sequencing Core Facility, National Health Laboratory Service, Johannesburg, South Africa

\section{Sabiha Yusuf Essack}

Antimicrobial Research Unit, College of Health Sciences, University of KwaZulu-Natal, Durban, South Africa

Ethical approval. Ethical approval was obtained from the University of KwaZulu-Natal Biomedical Research Ethics Committee (ref. no. BF512/16, sub-study of BCA444/16). Permission to conduct the research was also granted from the KwaZulu-Natal Department of Health, uMgungundlovu District and hospital managers.

Acknowledgements. We are grateful to the National Center for Biotechnology Information GENBANK submission staff for help with genome upload, decontamination and deposition procedures.

Author contributions. RCF co-conceptualised the study, undertook sample collection, microbiological laboratory and data analyses, prepared the table, interpreted results, contributed to bioinformatics analysis, and drafted the manuscript. LLF undertook sample collection and microbiological laboratory analyses, contributed to bioinformatics analysis and vetted the results. MA contributed to bioinformatics analyses. AI performed whole genome-sequencing analysis. SE co-conceptualised the study and undertook critical revision of the manuscript. All authors read and approved the final manuscript.
Funding. This work was supported by the Antimicrobial Research Unit and College of Health Sciences of the University of KwaZulu-Natal. The National Research Foundation (NRF) funded the study through NRF Incentive Funding for Rated Researchers (grant no. 85595), the NRF Competitive Grant for Rated Researchers (grant no. 106063) and the Department of Science and Technology/NRF South African Research Chair in Antibiotic Resistance and One Health (grant no. 98342). The South African Medical Research Council also funded the study through the Self-Initiated Research Grant. Any opinions, findings and conclusions or recommendations expressed are those of the authors and do not necessarily reflect the views of the organisations or agencies that provided support for the project. The funders had no role in the study design or the decision to submit the work for publication.

Conflicts of interest. SYE is chairperson of the Global Respiratory Infection Partnership and a member of the Global Hygiene Council, both sponsored by an unrestricted educational grant from Reckitt Benckiser. All other authors have no conflicts of interest.

1. Founou RC, Founou LL, Allam M, Ismail A, Essack SY. Whole genome sequencing of extended spectrum $\beta$-lactamase (ESBL)-producing Klebsiella pneumoniae isolated from hospitalized patients in KwaZulu-Natal, South Africa. Sci Rep 2019;9(1):6266. https://doi.org/10.1038/s41598-019$42672-2$

2. Kim JY, Song HS, Kim YB, et al. Genome sequence of a commensal bacterium, Enterococcus faecalis CBA7120, isolated from a Korean fecal sample. Gut Pathog 2016;8:62-62. https://doi.org/10.1186/ s13099-016-0145-x

3. Dai D, Wang $\mathrm{H}, \mathrm{Xu} \mathrm{X}$, et al. The emergence of multi-resistant Enterococcus faecalis clonal complex, CC4, causing nosocomial infections. J Med Microbiol 2018;67(8):1069-1077. https://doi. (10.1099/jmm 0.000761

4. Founou RC, Founou LL, Essack SY. Extended spectrum beta-lactamase mediated resistance in carriage and clinical gram-negative ESKAPE bacteria: A comparative study between a district and tertiary hospital in South Africa. ARIC 2018;7(1):134. https://doi.org/10.1186/s13756-018-0423-0

5. Song X, Sun J, Mikalsen T, Roberts AP, Sundsford A. Characterisation of the plasmidome within Enterococcus faecalis isolated from marginal periodontitis patients in Norway. PLoS ONE 2013;8(4):e62248. https://doi.org/10.1371/journal.pone.0062248

6. Soge OO, Beck NK, White TM, No DB, Roberts MC. A novel transposon, Tn6009, composed of a Tn916 element linked with a Staphylococcus aureus mer operon. J Antimicrob Chemother 2008;62(4):674-680. https://doi.org/10.1093/jac/dkn255

S Afr Med J 2021;111(2):98-99. https://doi.org/10.7196/SAMJ.2021.v111i2.15454 